\title{
Research on reasonable influence scope during road construction
}

\section{based on microscopic simulation}

\author{
Liang zijun ${ }^{1, a}$, Cao sen ${ }^{2, b}$ \\ ${ }^{1}$ Chang'an University, Nan'er Huan Road Xi'an, ShaanXi Province, China, 710064. \\ ${ }^{2}$ The Key Lab of Urban ITS Technology Optimization and Integration, Ministry of Public Security, \\ Huangshan Rd, Hefei, Anhui Province, China, 230088. \\ a953609319@qq.com, b903450291@qq.com.
}

Keyword: road construction, influence scope, microscopic simulation, delay, $\mathrm{t}$ test.

\begin{abstract}
In order to determine effectively the reasonable traffic influence range during city road construction, the microscopic simulation analyses the change of intersection delay, which is caused by road construction, taking five lanes in east - west and four lanes in south - north as study object, on the conditions of different saturation based on Vissim. According to compare intersection delay data before and after construction, this paper determines whether the road construction produces influences on the delay of vehicle in the intersection significantly, under the condition of $95 \%$ confidence interval, which applies t test, further more decides the reasonable influence scope of road construction. Compared with the influence scope of road construction, which is determined by Webster signal cycle method, it is found that the sensitivity of the determination method, which is based on $t$ test, for the influence scope of construction is higher, determining effectively the reasonable influence scope of road construction.
\end{abstract}

\section{Introduction}

In recent years, road construction in cities, not only make the traffic capacity of the road construction is affected, will also affect the surrounding road network. Reasonable research on the influence during road construction is one of the key factors on effectively traffic control ${ }^{[1]}$.

Some scholars study on traffic organization scheme during road construction, and evaluate the impact of the change of surrounding road network traffic duing road construction, by analysising on the influencing construction regional traffic status ${ }^{[2]}$. Some scholars use qualitative and quantitative analysis method to get the decision process and calculation method of the influence range of road construction. According to the capacity reduction rate of road traffic capacity, the influence of the construction is classified, and the threshold of traffic impact analysis is determined by the lowest limit of service level ${ }^{[3]}$. Domestic and foreign scholars confirm the influence range of road construction is determined by the optimal signal period of F. Webster -B.. based on analyzing the change of traffic capacity ${ }^{[5,6]}$. This paper analyzes the change of the intersection saturation caused by road construction, and discusses the calculation method of the related parameters of road construction. The data of intersection delay was obtained, by operating microscopic simulation software VISSIM, and the influence scope of road construction is determined by $\mathrm{t}$ test. 
2. The calculation method of parameters relevanted to influence scope of the road construction

\subsection{The relationship between saturation and road construction}

Calculating formula of saturation is the value of traffic flow divided by traffic capacity ${ }^{[7]}$ :

$$
x=q / C_{s} .
$$

Where: $x$-intersection saturation; $q$ - raffic flow; $C_{s}$ - traffic capacity of intersection.

\subsection{The relationship between road constuction and the signal cycle}

In order to further study the relationship between road construction and signal cycle, the paper introduces the method of Webster's method to establish the model of the period when the vehicle delay time is minimum ${ }^{[6]}$.

$$
\begin{aligned}
& C_{0}=\frac{1.5 L+5}{1-Y} . \\
& g_{i}=(C-L) \frac{y_{i}}{Y} .
\end{aligned}
$$

$L$ - total loss time of signal cycle(s), The loss time of each phase is 4 seconds, and the two phase signal is 8 seconds; $y_{i}$ - the maximum value of the v/s ratio of all the entrance lane during the phase

$i$, that is $y_{i}=\max \left\{(v / s)_{j}\right\}, Y$ - sum of all phases of the composition cycle, that is $Y=\sum y_{i}$, signal cycle should not be too long, If the calculated value $C$ is greater than 120 seconds, $\mathrm{C}$ takes 120seconds; and signal cycle is not too short, considering to the vehicle safety through the intersection to the shortest time and the shortest time of pedestrian crossing, If the calculated value $C$ is less than 40 seconds, $C$ takes 40 seconds.

\subsection{The relationship between road constuction and delay}

Theoretical delay calculation method is simplified formula for calculating the delay in Highway capacity Manual(HCM2010) ${ }^{[7]}$.

$$
d_{t, 1}=\min \left(d_{n m}, d_{m g}\right) .
$$

Vehicle delay without merging is shown as:

$$
\begin{aligned}
& d_{n m}=3600\left(\frac{1}{c_{n m}}-\frac{1}{1800}\right)+900 T\left[\frac{v_{1}}{c_{n m}}-1+\sqrt{\left(\frac{v_{1}}{c_{n m}}-1\right)^{2}+\frac{8 \mathcal{V}_{1}}{c_{m g}^{2} T}}\right] . \\
& d_{m g}=3600\left(\frac{1}{c_{m g}}-\frac{1}{1800}\right)+900 T\left[\frac{v_{m g}}{c_{m g}}-1+\sqrt{\left(\frac{v_{m g}}{c_{m g}}-1\right)^{2}+\frac{{ }^{8} \boldsymbol{V}_{m g}}{c_{m g}^{2} T}}\right]
\end{aligned}
$$

Where: $d_{n m}$ - delay of Single lane; $c_{n m}$ - Capacity of a single lane; $v_{1}$ - Total traffic flow. $d_{m g}{ }^{-}$ 
total delay; $c_{m g}$ - total traffic flow; $v_{m g}$ - total flow; $T$ - time of cycle.

\section{The determination method of road construction influence range}

\subsection{Saturation variation range and delay data}

Taking into account the saturation can not be greater than 1 , the change range of saturation is $[0$, $1]$, and the range of increased saturation is $[0,1]$. Saturation variation range as shown in Fig. 1.

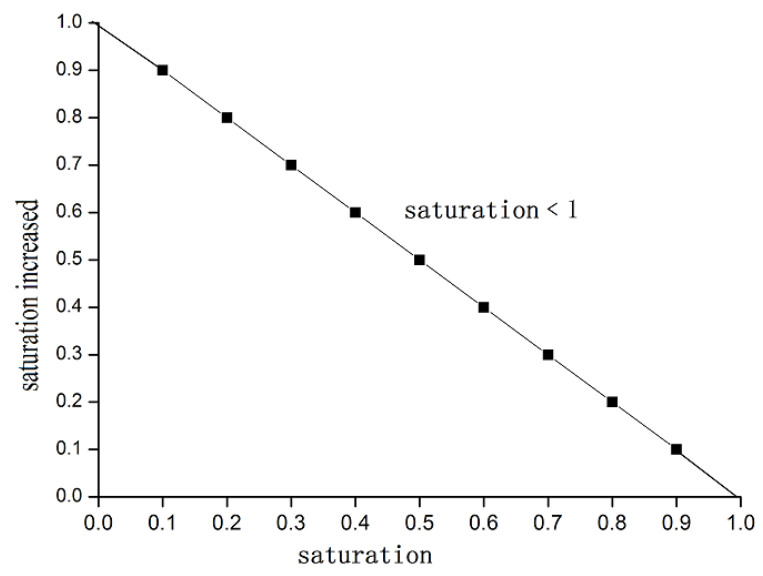

Fig. 1 the figure of saturation increased range

After determining the saturation range, the total time of the simulation is chosen. VISSIM simulation educe delay data sequence with different saturation. Assuming that saturation is 0.1 , the intersection delay data is expressed as: $A_{1}=\left\{a_{1}, a_{2}, \ldots, a_{n}\right\}$, without road construction; and $A_{1}^{\prime}=\left\{a_{1}^{\prime}, a_{2}, \ldots, a_{n}^{\prime}\right\}$, during road construction. Order by analogy, assuming that saturation is $\mathrm{x}$ the intersection delay data is expressed as: $A_{m}=\left\{a_{1}, a_{2}, \ldots, a_{n}\right\}$, without road construction; and $A_{m}^{\prime}=\left\{a_{1}^{\prime}, a_{2}, \ldots, a_{n}^{\prime}\right\}$, during road construction, $m=1,2 \ldots 9$. Average delay of intersection is $\bar{X}_{m}=\frac{a_{1}+a_{2}+\ldots+a_{n}}{n_{1}}$, without road construction; Average delay of intersection is $\bar{X}_{m}{ }^{\prime}=\frac{a_{1}^{\prime}+a_{2}{ }^{\prime}+\ldots+a_{n}^{\prime}}{n_{2}}$, during road construction. $n_{1}$ is the number of elements of $A_{m}$, and $n_{2}$ is the number of elements of $A_{m}^{\prime}$.

\subsection{Model of $t$ test}

In this paper, we take the $\mathrm{t}$ test to determine whether the delay sequence of $A_{1}$ and $A_{1}$ has a significant effect on the confidence interval of $95 \%$, so as to determine whether the results of the impact of road construction has a significant difference. Calculation formula of t test is shown as: 


$$
\begin{aligned}
& t=\frac{\bar{X}_{m}-\bar{X}_{m}}{\sqrt{\frac{\left(n_{1}-1\right) S_{1}^{2}+\left(n_{2}-1\right) S_{2}^{2}}{\left(n_{1}+n_{2}-2\right)}\left(\frac{1}{n_{1}}-\frac{1}{n_{2}}\right)}} . \\
& \left(d f=n_{1}+n_{2}-2\right) .
\end{aligned}
$$

Where: $s_{1}$ - the variance of $A_{m}, s_{2}$ - the variance of $A_{m}^{\prime}, d f$ - the freedom of $\mathrm{t}$ test. The significant effect was 1 , and no significant effect was set to 0 , There is a significant effect on the increase of the saturation of over 1 . The delay values of different saturation values are determined and expressed by the lower triangular matrix A with 9 rows and 9 columns.

\subsection{Webster signal cycle method}

Adjusting the signal timing to meet the traffic demand, and determining the scope of road construction, with applying Webster signal cycle formula. If the signal cycle is 1.2 times greater than the original signal cycle, the intersection is located Influence range of road construction. Calculation formula is shown as:

$$
C=\frac{1.5 L+5}{1-Y /(\omega \cdot \xi)} \geq 1.2 C_{0}
$$

Where: $Y \leq 0.9 ; C_{0}$ - original signal cycle; $\omega$ - order change factor of intersection. Order change factor of intersection is equal to the ratio of the traffic of non-motor vehicle, without road construction and during road construction. The influence range of road construction is obtained by formula calculation, expressed by the lower triangular matrix B with 9 rows and 9 columns.

\subsection{Comparison analysis of the influence scope of road construction}

Matrix $C$ is obtained by using matrix $A$ subtract matrix $B$. It is considered that there is a difference between the 0 results, when the element of matrix $C$ is not fully equal to 0 . The influence scope of road construction is more large and higher sensitivity, by using $t$ test, when the number of positive 1 is more than the number of negative 1 in matrix $C$, On the other hand, the versa. When the number of positive 1 and negative 1 is equal, it needs to be further analyzed.

\section{Case analysis}

\subsection{Intersection model}

Signal control intersections in the influence scope of road construction was taken as research object. East - west direction entrance road has 3 lanes: 1 straight lane, 1 straight right lane and 1straight left lane. North - south direction entrance road has 2 lanes: 1 straight right lane and 1straight left lane. Turn left car ratio is $15 \%$, turn right car ratio is $20 \%$ and straight car ratio is $65 \%$. Assuming that the traffic volume is determined according to the size of the saturation.

Road construction cause saturation change. With the increase of saturation, the signal cycle changes, and the traffic capacity of the intersection signal control is changed. The signal period and the capacity of different saturation corresponding to different saturation are calculated by the 
formula (1), (2) and (3).

Table 1 the relationship between saturation and signal cycle

\begin{tabular}{|c|c|c|c|c|c|c|c|c|c|}
\hline saturation & 0.1 & 0.2 & 0.3 & 0.4 & 0.5 & 0.6 & 0.7 & 0.8 & 0.9 \\
\hline cycle $(\mathrm{s})$ & 40 & 40 & 40 & 40 & 40 & 43 & 57 & 85 & 120 \\
\hline
\end{tabular}

Table 2 the relationship between signal cycle and capacity

\begin{tabular}{|c|c|c|c|c|c|c|c|c|c|}
\hline cycle (s) & 40 & 40 & 40 & 40 & 40 & 43 & 57 & 85 & 120 \\
\hline capacity (vehicle/h) & 2383 & 2383 & 2383 & 2383 & 2383 & 2418 & 2505 & 2593 & 2934 \\
\hline
\end{tabular}

\subsection{Delay data educed from VISSIM simulation}

Road construction cause traffic volume change. For the four entrance lanes, the average value of delay date obtained by 10 time continuous simulation, with the simulation time is 1200 seconds, the time interval is $60 \mathrm{~s}$, the delay data sequence is 10. Simulations are performed for different saturation values between 0.1 and 0.9 , and a set of data for the signal cycle is $40 \mathrm{~s}$ as shown in the following table 3 and table 4(other saturation data are obtained by VISSIM simulation).

Table 3 the table of delay data sequence without road construction(Unit is second)

\begin{tabular}{|c|c|c|c|c|c|c|c|c|c|}
\hline saturation & \multirow{2}{*}{0.1} & 0.2 & 0.3 & 0.4 & 0.5 & 0.6 & 0.7 & 0.8 & 0.9 \\
\hline 1 & & & & & & & & & \\
\hline 2 & 5.1 & 7 & 8.3 & 10.6 & 13.8 & 30.7 & 55 & 76.4 & 100.9 \\
\hline 3 & 6.3 & 11.8 & 10.6 & 2.9 & 18 & 10 & 78.8 & 75.9 & 122.5 \\
\hline 4 & 9.2 & 10.5 & 9.8 & 4.7 & 13.3 & 3.7 & 49.3 & 100.2 & 132 \\
\hline 5 & 0 & 5.5 & 8.6 & 8.9 & 12.4 & 27.9 & 74.7 & 107.3 & 94.2 \\
\hline 6 & 9.8 & 4.6 & 5.9 & 7.1 & 22.1 & 31.6 & 56.1 & 85.7 & 138.5 \\
\hline 7 & 3 & 9.4 & 10.2 & 7 & 17 & 35 & 73.6 & 110.6 & 145.3 \\
\hline 8 & 11.7 & 10.7 & 5.9 & 7.6 & 37.2 & 22.2 & 65.6 & 130.4 & 120 \\
\hline 9 & 5.9 & 9.8 & 10.3 & 8.5 & 26.9 & 27.9 & 77.8 & 110.7 & 121.4 \\
\hline 10 & 1 & 11.6 & 3.1 & 13.1 & 25.4 & 28.2 & 89.1 & 96.4 & 130 \\
\hline
\end{tabular}


Table 4 the delay data sequence during road construction(Unit is second)

\begin{tabular}{|c|c|c|c|c|c|c|c|c|c|}
\hline saturation & 0.1 & 0.2 & 0.3 & 0.4 & 0.5 & 0.6 & 0.7 & 0.8 & 0.9 \\
\hline 1 & & & & & & & & & \\
\hline 2 & 5.1 & 7 & 8.3 & 10.6 & 12.3 & 66.9 & 76.8 & 82.3 & 120.3 \\
\hline 3 & 6.3 & 11.8 & 10.6 & 2.9 & 8.1 & 70.1 & 82.4 & 108.6 & 125.9 \\
\hline 4 & 9.2 & 10.5 & 9.8 & 4.7 & 23.3 & 71.9 & 55.1 & 102.9 & 133.5 \\
\hline 5 & 0 & 5.5 & 8.6 & 8.9 & 26.9 & 74.4 & 74.7 & 99.7 & 106.9 \\
\hline 6 & 9.8 & 4.6 & 5.9 & 7.1 & $28 . .1$ & 56.9 & 76.3 & 98.6 & 138.5 \\
\hline 7 & 3 & 9.4 & 10.2 & 7 & 17 & 43.8 & $74 . .8$ & 112.7. & 158.9 \\
\hline 8 & 11.7 & 10.7 & 5.9 & 7.6 & 37.2 & 65.6 & 73.1 & 125.3 & 147.4 \\
\hline 9 & 5.9 & 9.8 & 10.3 & 8.5 & 26.9 & 77.7 & 82.4 & 120.7 & 132.3 \\
\hline 10 & 1 & 11.6 & 3.1 & 13.1 & 35.4 & 69.9 & 92.7 & 109.6 & 136.9 \\
\hline
\end{tabular}

\subsection{Result and comparative analysis}

1. Matrix $A$ represent the influence scope of road construction obtained from t test, with delay data of VISSIM microscopic simulation and formula (7).

2. Matrix $B$ represent the influence scope of road construction obtained from Webster 1.2 times cycle, with formula (8) and table 1.

3. Matrix $C$ is the result of matrix $A$ subtract matrix $B$.
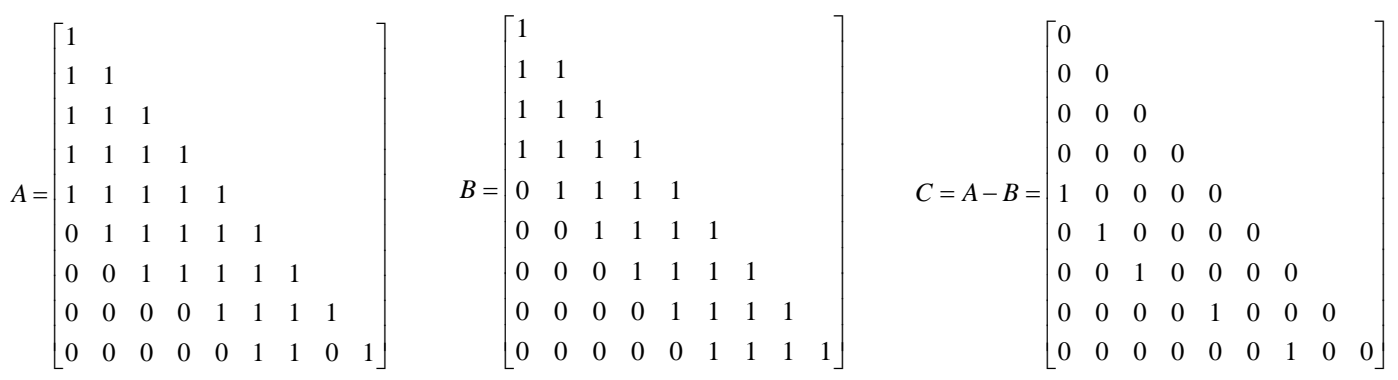

There are five positive 1 and no negative 1 in matrix $C$. It is show that the results of the $t$ test model are more larger and sensitive than Webster's 1.2 times cycle in the scope of influence, because the number of positive 1 is more than the number of negative 1 .

\section{Conclusion}

In this paper, the impact of urban road construction on the intersection was transferred on saturation, and explore the relationship between road construction and saturation, signal cycle and delay. The delay data is obtained from VISSIM simulation without road construction and during road construction. It is adopting $t$ test that judge whether or not the significant influence on the delay data. The comparative analysis originate from comparing matrix A with matrix B. It is show that the results of the t test model are more sensitive than Webster's 1.2 times cycle in the scope of influence in case analysis. In the practical application, this study can more accurately analyze the delay data changes caused by road construction, so as to provide the basis for the traffic managers to make effective decisions. 


\section{Reference}

[1] Guo xiaofeng, Li zhigang. Urban roadway taking-up construction traffic impact analysis and countermeasure research[J]. Urban Roads Bridges \& Flood Control, 2008, (6): 93-96.

[2] JIANG Y.Traffic Characteristics and Estimation of Traffic Delays and User Costs at Indiana Freeway Work Zone[J].Transportation Research Record, 1999, 16571.

[3] Xie tiejian, Lin hangfei, Han shengfeng. Research on the traffic organization of regional large-scale road construction -- a case study of Shanghai World Expo supporting road construction traffic organization[J]. Traffic \& Transportation, 2007, 42 (4).

[4] Zhou tao,An meng,Zhai changxu. The traffic organization research during urban road construction[J]. Journal of Transportation Engineering and Information. 2012, 10(1):6-12.

[5] Zhang weihua, Huang wenjuan, Ding heng, et al. Construction of urban road traffic organization optimization theory and key technology research[D]. He fei: Transportation Research Institute of Hefei University of Technology, 2008.

[6] Ma xiao, Ding heng, Zhang weihua, et al. Research on the method of determining the influence range of road construction[J]. Journal of Hefei University of Technology(Natural Science), 2011, 34 (5) : 655-660.

[7] Transportation research board. Highway capacity Manual(HCM)special report 209. Washington D.C.[s.n.], 2010. 